\title{
Flow Cytometric Measurement of Aneuploid DNA Content Correlates with High S-Phase Fraction and Poor Prognosis in Patients with Non-Small-Cell Lung Cancer
}

\author{
Rancés Blanco, ${ }^{1}$ Charles E. Rengifo, ${ }^{2}$ Mercedes Cedeño, ${ }^{1}$ \\ Milagros Frómeta, ${ }^{1}$ and Enrique Rengifo ${ }^{1}$ \\ ${ }^{1}$ Laboratory of Specific Recognition and Biological Activity, Department of Quality Control, Center of Molecular Immunology, \\ 216 Street and 15 Avenue, Atabey, Playa, P.O. Box 16040, 11600 Havana, Cuba \\ ${ }^{2}$ Department of Pathology, Manuel Fajardo General Hospital, Zapata and D Street Vedado, Plaza de la Revolución, \\ 10400 Havana, Cuba
}

Correspondence should be addressed to Rancés Blanco; rances@cim.sld.cu

Received 21 June 2013; Accepted 18 July 2013

Academic Editors: L. Daniele, M. Jensen, and Z. Wang

Copyright (c) 2013 Rancés Blanco et al. This is an open access article distributed under the Creative Commons Attribution License, which permits unrestricted use, distribution, and reproduction in any medium, provided the original work is properly cited.

\begin{abstract}
Cellular DNA content (ploidy) and proliferation activity (e.g., S-phase fraction) measured by flow cytometry have been usually related to the biologic aggressiveness of various neoplasms. In this study, these parameters were analyzed in paraffin-embedded tumor specimens from 43 cases of resected non-small-cell lung cancer (NSCLC). Additionally, the correlation of them with both prognosis and a variety of clinic-pathological features were investigated. The stage and the appearance of both local recurrence and metastasis were related to overall survival of patients. Twenty-two tumors (51.2\%) had a diploid DNA distribution, while 21 were aneuploid (48.8\%). The mean of aneuploidy was $1.6 \% \pm 0.3 \%$. A correlation was found between ploidy and survival as well as with the appearance of local recurrence and/or metastasis. The mean values of S-phase fraction of diploid and aneuploid tumors were $16.7 \pm 11.3 \%$ and $32.9 \pm 12.1 \%$, respectively, which were significantly different $(P=0.0022)$. Similar results were obtained analyzing the proliferation index (sum of cells in $\mathrm{S}$ and $\mathrm{G}_{2} / \mathrm{M}$ phases of cell cycle) $(P=0.0040)$. However, no correlation between these parameters and both overall survival of patients and clinicopathological features was observed. Our results could suggest the potential use of ploidy analysis as a useful complement of TNM stage in NSCLC.
\end{abstract}

\section{Introduction}

Lung cancer is one of the most frequently occurring neoplasms and is the leading cause of death by cancer worldwide [1]. There are two main variants of the disease, non-smallcell lung cancer (NSCLC) and small cell lung cancer (SCLC). However, NSCLC represents more than $80 \%$ of all lung carcinomas [2].

Usually patients with NSCLC have a poor prognosis because most of them present with advanced or metastatic diseases at the time of diagnosis. It has been estimated that only $10-15 \%$ of the patients will ultimately be cured [3]. In patients with NSCLC, some genetic and regulatory aberrations have been considered responsible for the tumor survival advantage. For that reason, several tumor markers, clinic-pathological indicators, and genetic alterations have been assessed in NSCLC for identifying patients with a poor prognosis and for a better guide of treatment strategy [4].

It is known that the development of malignant neoplasm occurs through a multistep genetic evolution process which often is accompanied by chromosome instability $[5,6]$. Chromosomal instability of tumors leads to cell clones and to numerical and structural chromosome abnormalities. These changes in the number of whole chromosomes (aneuploidy) can stimulate oncogenes and cause amplification and overexpression of them [7].

Some investigations regarding the prognostic value of DNA content (ploidy) and S-phase fraction (percentage of cells in S-phase of cell cycle) have been greatly facilitated by the application of flow cytometry using nuclei isolated from 
paraffin-embedded tissues [8]. In this sense, flow cytometry permits a rapid, accurate, and quantitative evaluation of DNA content in large numbers of individual nuclei isolated from solid human neoplasms [9].

Ploidy status and S-phase fraction (SPF) analyzed by flow cytometry have been established as useful indicators for the biologic aggressiveness of various neoplasms [10, 11]. However, in NSCLC patients the values of ploidy and SPF as reliable biomarkers still remain controversial [5, 12-14]. The aim of this study was to evaluate the relationship between DNA ploidy, SPF, proliferation index (sum of cells in $S$ and $\mathrm{G}_{2} / \mathrm{M}$ phases), and some clinicopathological parameters as well as their impact in the overall survival of NSCLC patients.

\section{Materials and Methods}

2.1. Tissue Specimens. A number of 43 routinely-processed, formalin-fixed, and paraffin-embedded archival samples with diagnosis of NSCLC were received from the pathology department of both Hermanos Ameijeiras General Hospital and the National Institute of Oncology and Radiobiology after the approved consent by the institutional ethical committees.

2.2. Pathological Features. The evaluation of some morphological parameters such as histopathological classification, grade of differentiation, degree of cell pleomorphism, and necrosis index was performed for an expert pathologist (Charles E. Rengifo) in each tumor tissue using hematoxylin and eosin (H/E) staining as it was previously described in [15]. Briefly, differences in some cytomorphologic characteristics such as cell and nuclear sizes, cellular shape, chromatin pattern, nucleoli, and amount of cytoplasm were considered to express the degree of cell pleomorphism which was scored as follows: low, moderate and high cell pleomorphism. Concerning the degree of tumor necrosis (necrosis index), a low-power field with 100x magnification (10x ocular, 10x objective) of each section was evaluated and it was scored subjectively as follows: low (less than $50 \%$ of necrosis areas per field) and high (more than $50 \%$ of necrosis areas per field). All analyses were performed using an Olympus BX51 brightfield microscope.

2.3. Quality Evaluation of Sample Preparation. Before nuclei isolation from formalin-fixed and paraffin-embedded sections, regions of tissues with an abundant number of tumor cells were carefully chosen using a conventional light microscope (Olympus BX51) based on hematoxylin and eosin staining. Tissue areas with abundant tumor necrosis and/or lack of habitual morphology were selected and marked in each histological slide, transferred to the corresponding paraffin block, and removed from it.

After nuclei isolation and propidium iodide staining (see Section 2.4), each final preparation (nuclei suspension) was checked under a conventional fluorescent microscope (Leica DMRXA). Samples with clumping or excess debris, presence of abundant cytoplasmic remnants attached to nuclei, and/or an elevate number of nuclei with morphological appearance of nontumoral were eliminated and these samples were reprepared.
2.4. DNA Content, $S$ and $G_{2} / M$ Phases Measurements. The flow cytometry methodology using nuclei isolated from formalin-fixed and paraffin-embedded tissues, as was previously described [16] with some modifications, was used. Briefly, two or three $50 \mu \mathrm{m}$ sections from each tissue block were dewaxed in xylene, rehydrated in ethanol, and finally washed in distilled water. Afterward, the tissues were treated with $0.2 \%$ citrate acid pH6 solution for $2 \mathrm{~h}$ at $80^{\circ} \mathrm{C}$ and then with $0.5 \%$ pepsin solution (Sigma P7012) $(\mathrm{pH} 1.5)$ at $37^{\circ} \mathrm{C}$ for 10 minutes. Single-cell suspensions were filtered through $50 \mu \mathrm{m}$ nylon mesh and routinely washed. Dissociated nuclei were adjusted to a final concentration of $1 \times 10^{6}$ nuclei/mL and were then stained with propidium iodide (Sigma P4170) at a final concentration of $40 \mu \mathrm{m} / \mathrm{mL}$. A number of 10.000 nuclei were acquired for each sample, at a low flow rate, using an Ortho Cytoron Absolute flow cytometer (Ortho Diagnostic Systems, Tokyo, Japan) equipped with a $15 \mathrm{MW}$ argon laser. The data obtained was analyzed using the ModFit LT software (DNA Modeling System) version 2.0 (Verity Software House, Inc.) and single parameter histograms.

Histogram was considered unsuitable for interpretation if its variation coefficient $\mathrm{CV}$ (breadth of the fluorescence signal for normal cell population in $G_{0} / G_{1}$ phase at the middle of its height) exceeded 10\%. A sample was considered DNA diploid if, on the histogram, there was a single peak in the $G_{0} / G_{1}$ phase. DNA aneuploidy was defined if there was at least one separate second $G_{0} / G_{1}$ population to the right of the first $\mathrm{G}_{0} / \mathrm{G}_{1}$ peak. The ploidy of each tumor was characterized by the DNA index (DI: DNA tumor cells/DNA control cells) and the type of DNA content histogram (diploid: DI $=1$, aneuploid: $\mathrm{DI}<$ or $>1$ ). Tetraploid and multiploid histograms were classified into the aneuploid group.

The SPF and the percentage of cells in $G_{2} / M$ phase were calculated from each DNA histogram. In case of aneuploid histograms, percentage of cells in $S$ and $G_{2} / M$ phases was only shown for aneuploid population. The SPF values were divided into two groups; a low-SPF group defined as having SPF less than $25 \%$ of proliferating cells and a high-SPF group with SPF greater than $25 \%$. The proliferation index [12] was calculated as the sum of cells in $S$ and $G_{2} / M$ phases of cell cycle and was scored as it was previously described for SPF.

2.5. Statistical Analysis. GraphPad Prism 5 software (2007 GraphPad Software Inc., La Jolla, CA, USA) was used for data analysis. Where appropriate, the data were expressed as mean \pm standard deviation (SD). Survival distribution was estimated by the Kaplan-Meier method. Two-sided log-rank tests were performed to compare overall survival (OS) distribution according to DNA content, SPF, proliferation index, as well as the clinic-pathological features. Chi-square and Fisher's exact tests were used to compare groups and parameters. The correlation between SPF and proliferation index was assessed by Spearman ranks correlation coefficients. For all tests, the criterion for statistical significance was $P<0.05$.

\section{Results}

3.1. Patient Description and Pathological Features. Tables 1 and 2 showed a summary of patient characteristics and some 
TABLE 1: Patients characteristics.

\begin{tabular}{lc}
\hline Features & No. $(\%)(n=43)$ \\
\hline Gender & \\
Female & $15(34.9)$ \\
Male & $28(65.1)$ \\
Age (years) & \\
$\quad<60$ & $31(72.1)$ \\
$60-70$ & $9(20.9)$ \\
$>70$ & $3(7.0)$ \\
Tumor stage & \\
I & $22(51.2)$ \\
II & $8(18.6)$ \\
III A & $5(11.63)$ \\
III B & $1(2.3)$ \\
IV & $7(16.3)$ \\
Tumor size (cm) & \\
$<3$ & $10(23.3)$ \\
$\geq 3$ & $33(76.7)$ \\
Recurrence & \\
Yes & $8(18.6)$ \\
No & $35(81.4)$ \\
Metastasis* & \\
Yes & $15(36.6)$ \\
No & $26(63.4)$ \\
Overall survival & \\
Alive & $29(67.4)$ \\
Dead & $14(32.6)$ \\
Median (months) & 58.4 \\
\hline &
\end{tabular}

No.: number of cases; \%: percentages; ${ }^{*} n=41$.

TABLE 2: Tumor characteristics.

\begin{tabular}{lc}
\hline Features & No. $(\%)(n=43)$ \\
\hline Histopathological type & \\
$\quad$ Squamous cell carcinoma & $14(32.5)$ \\
Adenocarcinoma & $16(37.2)$ \\
Large cell carcinoma & $7(16.3)$ \\
Other & $6(14.0)$ \\
Grade of differentiation & \\
$\quad$ Well & $8(18.6)$ \\
Moderate & $15(34.9)$ \\
Poor & $20(46.5)$ \\
Degree of cell pleomorphism & \\
Low & $17(39.5)$ \\
Moderate & $16(37.2)$ \\
High & $10(23.3)$ \\
Necrosis index & \\
$<50 \%$ & $31(72.1)$ \\
$\geq 50 \%$ & $12(27.9)$ \\
\hline
\end{tabular}

No.: number of cases; \%: percentages.

pathological features. The gender ratio was close to $2: 1$ in favor of males, with a mean age of 56.9 years (range 4576 years). Indistinctly, lymph node and other site metastases were evidenced in 15/41 (36.6\%) patients and 26/41 (63.4\%) of them have not shown any metastatic site. In
TABLE 3: Distribution of S-phase fraction and proliferation index according to DNA content status.

\begin{tabular}{lcccc}
\hline Factor & $\begin{array}{c}\text { All patients } \\
(n=43)\end{array}$ & $\begin{array}{c}\text { Diploid } \\
(n=22)\end{array}$ & $\begin{array}{c}\text { Aneuploid } \\
(n=21)\end{array}$ & $P$ value \\
\hline $\begin{array}{l}\text { S-phase fraction } \\
\text { (SPF) }\end{array}$ & & & & \\
$\quad$ Low & 23 & 17 & 6 & 0.0022 \\
$\quad$ High & 20 & 5 & 15 & \\
\hline $\begin{array}{l}\text { Proliferation } \\
\text { index }\end{array}$ & & & & \\
$\quad$ Low & 16 & 13 & 3 & 0.0040 \\
$\quad$ High & 27 & 9 & 18 & \\
\hline
\end{tabular}

SFS: cell in S-phase of cell cycle, proliferation index: cells in both $S$ and $G_{2} / M$ phases of cell cycle, low: less than $25 \%$ of proliferating cells, high: more than $25 \%$.

general, 14/43 (32.5\%) patients died, and the median overall survival of the population was 58.4 months (ranged from 1.0 to 91.3).

3.2. Stage of the Disease, Local Recurrence, and Occurrence of Metastasis Were Significantly Predictive of Survival Outcome. The stage (I-IIIA versus IIIB-IV) was found to be associated with the overall survival of NSCLC patients $(P=0.0002$; log-rank test) (Figure 1(a)). In addition, the appearance of both recurrence and metastasis was related to overall survival of patients $(P=0.0044$ and $P=0.0002$, respectively, log-rank test) (Figures 1(b) and 1(c)). Differences in sex, age at diagnosis, tumor size, histopathological type, grade of differentiation, degree of cell pleomorphism, and index of tumor necrosis were not significantly predictive of survival outcome.

3.3. Aneuploidy Was Found in about the Half of NSCLC. Adequate DNA histograms were evidenced in all samples with a mean coefficient of variation of $7.5 \pm 2.2 \%$. DNA diploidy was detected in $22(51.2 \%)$ tumors and DNA aneuploidy (including tetraploidy and multiploidy) was detected in 21 tumors (48.8\%). The mean of DNA aneuploidy was $1.6 \% \pm$ $0.3 \%$ (Table 3). Median overall survival of the population was 66.5 months (ranged from 23.0 to 91.3) for patients with diploid tumors and 49.9 months (ranged from 1.0 to 90.5 ) for patients with aneuploid tumors. The aneuploid DNA content status was found to be associated with a poor overall survival of NSCLC patients ( $P=0.0390$; log-rank test) (Figure $1(\mathrm{~d})$ ).

3.4. Aneuploid Tumors Displayed Both Higher S-Phase Fraction and Proliferation Index. Low SPF and proliferation index were found in 23/43 (53.5\%) and 16/43 (37.2\%) tumors; respectively, while 20/43 (46.5\%) and 27/43 (62.3\%) samples displayed higher SPF and proliferation index, respectively. Overall, the mean of SPF of the tumors was $24.6 \pm 14.2 \%$. But, the mean values of SPF of the DNA diploid and aneuploid tumors were $16.7 \pm 11.3 \%$ and $32.9 \pm 12.1 \%$, respectively, which was significantly different $(P=0.0022$; Fisher's exact test) (Table 3) (Figure 2). Concerning proliferation index, the mean values were $26.3 \pm 12.1 \%$ and $40.1 \pm 14.2 \%$ for diploid and aneuploid tumors, respectively, which was also 


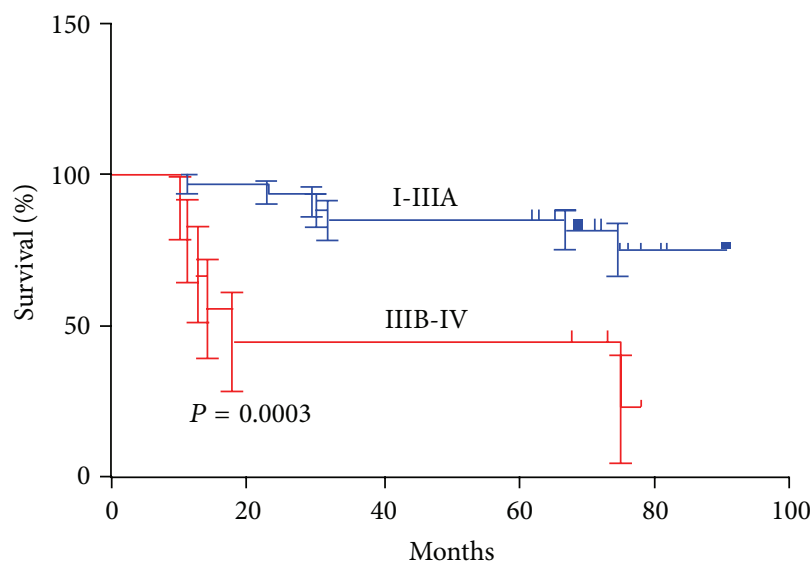

(a)

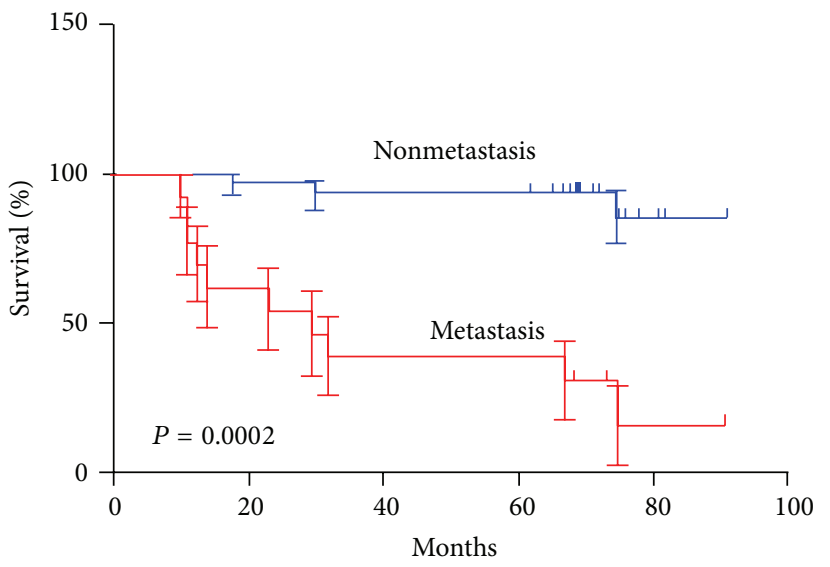

(c)

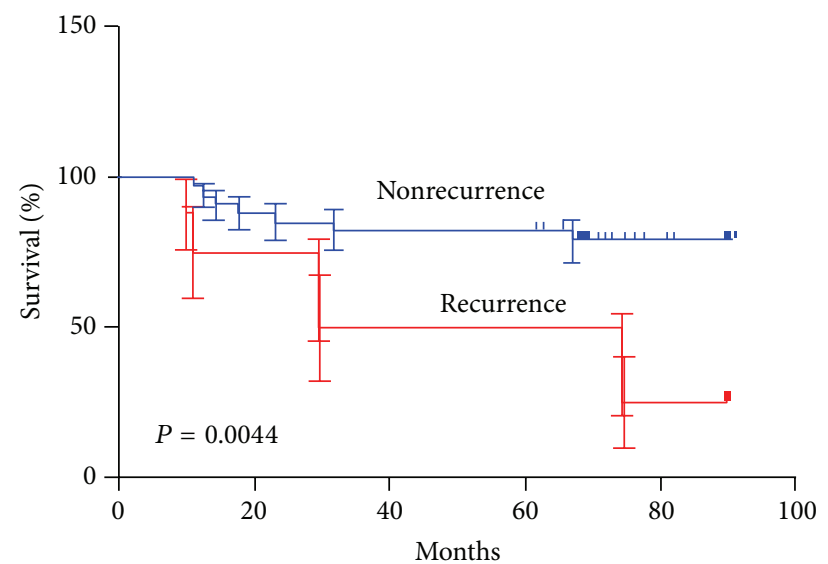

(b)

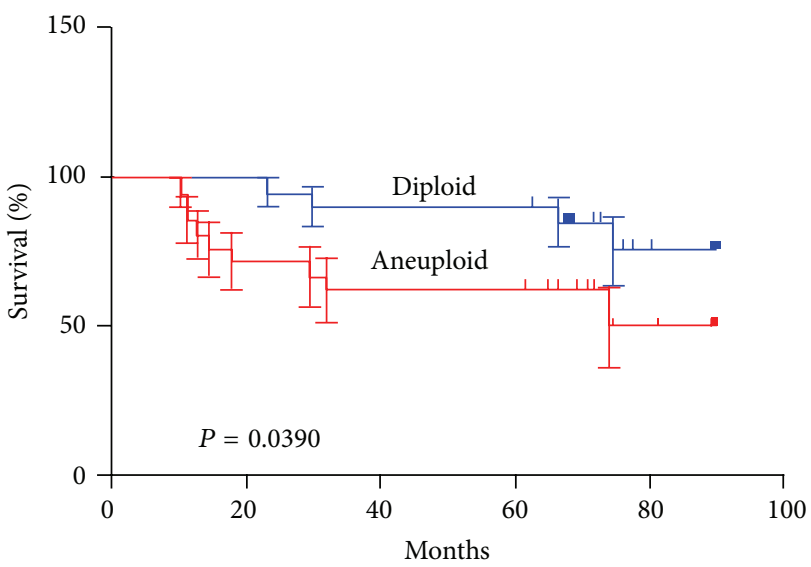

(d)

Figure 1: Comparison of overall survival of patients according to stage of the disease (a), local recurrence (b), lymph node and/or other site metastasis (c), and DNA content (d). Survival distribution was estimated by the Kaplan-Meier method, and two-sided log-rank tests were performed. The criterion for statistical significance was $P<0.05$.

significantly different $(P=0.0040$; Fisher's exact test $)$. A significant correlation was detected when the SPF and proliferation index of tumors were compared $(P<0.0001$, $\mathrm{rs}=0.7178$; Spearman test). However, no correlation between the both parameters and the overall survival of patients was observed.

3.5. DNA Ploidy Correlates with the Appearance of Local Recurrence and/or Metastasis. In the group of patients with aneuploid tumors, the appearance of local recurrence and/or metastasis was significantly more frequent $(9 / 21 ; 42.8 \%)$ than that in patients with diploid tumors $(6 / 22 ; 27.3 \%)(P=$ 0.0169; Fisher's exact test). There were no differences in the frequencies of DNA content status, SPF, and proliferation index when gender, age of patients, pathologic stage, tumor size, recurrence, occurrence of metastasis, histopathological type, grade of differentiation, degree of cell pleomorphism, and index of tumor necrosis were considered.

\section{Discussion}

Disease stage and performance status at time of diagnosis have been considered the two most noticeable prognostic factors in patients with NSCLC [3]. The TNM (tumor, node, and metastasis) staging system also leads the clinicians to the selection of a more appropriate conventional treatment (e.g., surgery, radiation, or chemotherapy) for NSCLC patients [17]. In this study, we showed an increased overall survival of patients at stages I-IIIA as compared with that of the group of patients at stages IIIB-IV, as well as that in patients with absence of metastasis (lymph node and other sites) and local recurrence. Nevertheless, the pathology-based TNM stage classification has been considered to provide imprecise information about the survival rates [18]. In consequence, numerous studies are currently focusing on the evaluation of other biological and molecular prognostic factors as a potential complement of TNM staging system $[12,19,20]$.

The biological behavior of a lung carcinoma is affected primarily by genetic abnormalities of the malignant cells [5]. The aberrations in the number of whole chromosomes (aneuploidy) and imbalances induced by chromosomal instability have been considered an important factor in lung carcinogenesis, responsible for tumor progression [21]. In lung cancer, DNA content abnormalities have been described as a heterogeneous spectrum in DNA histogram patterns of impaired tumor cell [22]. Aneuploid DNA content has been 


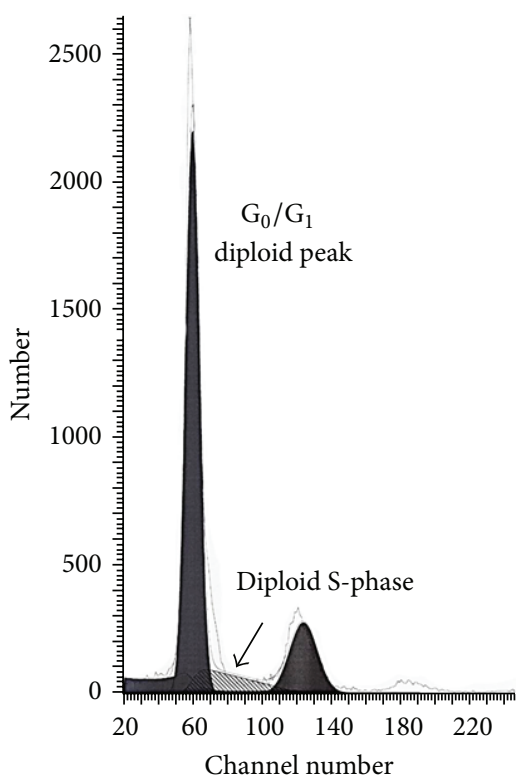

(a)

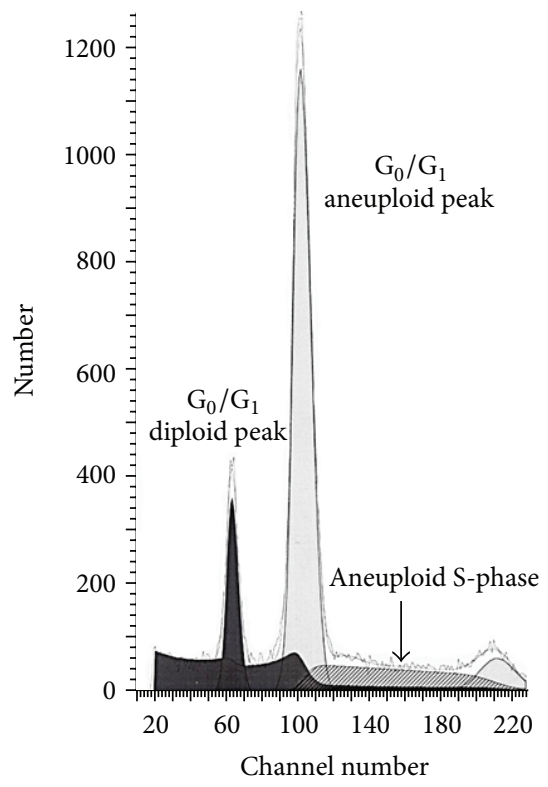

(b)

FIGURE 2: DNA content (ploidy) analysis in non-small-cell lung carcinoma samples measured by the flow cytometric profile and analyzed by ModFit LT software. Cells prepared by the Hedley method [16] and stained with propidium iodide. (a) DNA histogram of diploid tumor. Note that a single $G_{0} / G_{1}$ population. (b) DNA histogram of aneuploid tumor. Observe the appearance of one separate second $G_{0} / G_{1}$ population to the right of the first $G_{0} / G_{1}$ peak. The percentage of cells in S-phase of cell cycle was calculated from each DNA histogram. In case of aneuploid histograms, percentage of cells in S-phase was only shown for aneuploid population. Note that DNA aneuploid tumors have higher SPF than DNA diploid tumors do $(P=0.0022$; Fisher's exact test $)$.

found between $44.5-84.8 \%$ of NSCLC using flow cytometric analysis from archival formalin-fixed and paraffin-embedded specimens $[12,14,23,24]$.

In the present work, we observed the aneuploid DNA content in about half of the NSCLC samples. Similar to other reports, no differences between DNA content and gender, age of patients, pathologic stage, tumor size, grade of differentiation, degree of cell pleomorphism, and index of tumor necrosis were obtained $[23,25]$. Nevertheless, the aneuploidy was found to be associated with a poor overall survival of NSCLC patients, not depending on the histopathological subtype of tumor. It is known, aneuploidy has been usually related to an aggressive biological behavior of many malignant tumors [26] including lung cancers [12, 21, 23, 25]. In previous reports, other authors have obtained significant differences dependent on ploidy in both overall survival and disease-free survival in NSCLC $[12,23]$. However, in the majority of these studies, only squamous cell carcinoma was included, probably to avoid the potential impact of histologic type of the tumor on the survival rate $[23,25]$ or this subtype predominated in the series [12].

In aneuploid NSCLC, a greater tendency to displayed local recurrence, lymph node and/or distant metastasis was noted as compared with diploid tumors. In similar reports, more patients with aneuploid cancers died from distant metastasis, whereas local cancer recurrence was more frequent in patients with diploid tumors [23]. Additionally, in aneuploid NSCLC, a greater tendency to invade lymphatic vessels and more metastasis in regional lymph nodes were evidenced [12]. The appearance of local recurrence and/or metastasis in aneuploid tumors seems to be related to the substantial worse overall survival of these patients. In previous works, substantial differences in survival expectancy were observed in relation to the occurrence of metastasis in regional lymph nodes and local recurrence $[23,25]$.

At present, more research efforts are focusing on the evaluation of a variety of molecular disorders (e.g., protooncogene activation or mutations of suppressor and mutator genes) in order to determine the genetic profile connected with a high risk of recurrence of the disease after a radical surgical treatment than on DNA content analysis [23]. It could be related to the fact that the importance of DNA content status as a reliable prognostic marker in NSCLC still remains controversial [5, 12-14]. The substantial divergence of opinion concerning the prognostic value of DNA ploidy has been related to many causes such as the existence of diploid and aneuploid regions within a single-sample (tumor heterogeneity), nonstandard conditions of ploidy assessment and poor quality of tumor tissue for the cytometric analysis [23].

Here, hematoxylin and eosin staining permitted to select potential low-quality regions of each sample and/or paraffin blocks from several ones by the pathologist on the basis of histological slides, transferring these areas to each paraffin block and removed. This procedure allows the selection of regions with higher number of tumor cells, minimizing the possibility of significant mixture of nonepithelial cells and necrotic areas and, therefore, enhancing the quality of samples preparation. Similar technique in order to improve the quality of DNA analysis was previously reported by other 
authors [12]. However, the use of a bivariate flow cytometric measure combining cytokeratin and DNA staining to exclude nonepithelial cells from DNA analysis should be evaluated by our group.

In the present study, it was found that non-small-cell lung carcinomas with aneuploid DNA have higher proliferative activities than DNA diploid tumors, measured by flow cytometry. Aneuploid tumors displayed a significantly higher proportion of cells in SPF of cell cycle and, correspondingly, a lower proportion of $G_{0} / G_{1}$ phase cells than those displayed by tumors with DNA diploidy, similar to previous reports [27-30]. Moreover, in DNA aneuploid tumors the sum of cells in $S$ and $G_{2} / M$ phases of cell cycle (proliferative activity index) was also significantly higher as compared with diploid tumors. In previous works, the relationship between DNA content and distributions of the cell cycle phases has been demonstrated. Furthermore, an association between DNA content and Ki-67 expression has been reported [31]. However, Ki-67 is an antibody that recognizes the nuclear antigen not expressed during the $\mathrm{G}_{0}$ phase, but this Mab is reactive during the resting $\left(G_{1}, S, G_{2}\right.$, and $\left.M\right)$ phases of the cell cycle. Consequently, the proliferative activity index used in this study could offer a more precise piece of information about the factions of cells in $S$ and $\mathrm{G}_{2} / \mathrm{M}$ phases of cell cycle. However, other studies found that there were no differences in proliferative activity between the DNA ploidy patterns [14, 32-34].

In previous studies, the percentage of cells in S phase of cell cycle had been considered an independent, unfavorable prognostic factor in patients with squamous cell lung carcinoma [12] and adenocarcinoma [24]. However, here, we obtained no differences in the prognosis of patients with high and low cell proliferation neither with tumor size nor with the ability of these tumors to develop local recurrence and metastasis. These results could suggest that the increasing proliferative activity of tumors is probably not the only factor related to a major risk of recurrence and metastasis in NSCLC patients. In line with our results, the significance of SPF as a reliable predictor of recurrence has been questioned despite its correlation with the tumor growth rate [26]. In addition, it has been also suggested that SPF is not directly associated with the ability of a tumor to metastasize [26]. Nevertheless, to support whether there is any difference in the proliferative activity between DNA diploid and aneuploid tumors, as well as to assess the potential relation between proliferative activity and tumor size and local recurrence and metastasis, a large-scale study will be necessary.

Although flow cytometry is considered a powerful, rapid, and cost-effective technique for the identification and monitoring of diverse human diseases, nowaday this highthroughput technology has not been widely used except in a minority of specialized research institutions. However, the analysis of cellular DNA content measured by flow cytometry is a relatively easy test [25] and it has the potential to provide benefits through improved understanding of disease pathogenesis, identification of a subpopulation of NSCLC patients with both poor overall survival $[12,23]$ and high risk of recurrence and/or metastasis and in the development and design of specific therapies $[23,25]$. These piece of evidences, together, make the evaluation of ploidy status and proliferative activity of solid tumors by flow cytometry feasible to use as a routine laboratory procedure, not only in large medical centers but also in medium-sized hospitals and other professional reference centers. In spite of the fact that flow cytometers are generally instruments expensive to purchase and maintain, our results could suggest the potential use of DNA ploidy as a valuable complement of TNM stage in NSCLC as well as the introduction of this analysis into standard clinical practice $[12,23]$.

\section{Conclusions}

In summary, we reported the DNA aneuploid content in NSCLC patients using flow cytometric analysis from archival formalin-fixed, paraffin-embedded specimens. The quality of DNA analysis was improved on the basis of histological slides using optical microscopy. The correlation of aneuploidy with poor prognosis of patients as well as with the appearance of local recurrence and/or metastasis was also demonstrated. Additionally, we showed that DNA aneuploid tumors have both higher SPF and proliferative activity index than DNA diploid tumors. However, no correlation between these parameters and the overall survival of patients nor with the rest of the clinic-pathological features was obtained. Our results could suggest the potential use of DNA ploidy analysis as a valuable complement of TNM stage in NSCLC as well as the introduction of this analysis into standard clinical practice. A bivariate flow cytometric measure combining cytokeratins and DNA staining and a large-scale study are recommended.

\section{Conflict of Interests}

The authors report no conflict of interests.

\section{Authors' Contribution}

Rancés Blanco and Charles E. Rengifo contributed equally to this work.

\section{Acknowledgment}

Financial support was provided by the Center of Molecular Immunology.

\section{References}

[1] K. Kaira, N. Oriuchi, N. Sunaga, T. Ishizuka, K. Shimizu, and N. Yamamoto, "A systemic review of PET and biology in lung cancer," American Journal of Translational Research, vol. 3, no. 4, pp. 383-391, 2011.

[2] G. Giaccone and P. A. Zucali, "Src as a potential therapeutic target in non-small-cell lung cancer," Annals of Oncology, vol. 19, no. 7, pp. 1219-1223, 2008.

[3] T. Berghmans, M. Paesmans, and J.-P. Sculier, "Prognostic factors in stage III non-small lung cancer: a review of conventional, 
metabolic and new biological variables," Therapeutic Advances in Medical Oncology, vol. 3, no. 3, pp. 127-138, 2011.

[4] E. Blanchard, "Targeted agents in non small cell lung cancer," Cancer Therapy, vol. 6, pp. 95-102, 2008.

[5] K. Ueda, Y. Kaneda, M. Hayashi, M. Jinbo, T.-S. Li, and K. Hamano, "Relationship among genetic alterations, DNA content, and clinicopathological features in primary lung adenocarcinomas," Cancer Genomics and Proteomics, vol. 3, no. 3-4, pp. 265-270, 2006.

[6] C. R. Thoma, A. Toso, P. Meraldi, and W. Krek, "Mechanisms of aneuploidy and its suppression by tumour suppressor proteins," Swiss Medical Weekly, vol. 8, no. 141, article w13170, 2011.

[7] D. J. Gordon, B. Resio, and D. Pellman, "Causes and consequences of aneuploidy in cancer," Nature Reviews Genetics, vol. 13, no. 3, pp. 189-203, 2012.

[8] G. P. M. Ten Velde, B. Schutte, A. Vermeulen, A. Volovics, M. M. J. Reynders, and G. H. Blijham, "Flow cytometric analysis of DNA ploidy level in paraffin-embedded tissue of non-small-cell lung cancer," European Journal of Cancer and Clinical Oncology, vol. 24 , no. 3, pp. 455-460, 1988.

[9] W. E. Corver and C. J. Cornelisse, "Flow cytometry of human solid tumours: clinical and research applications," Current Diagnostic Pathology, vol. 8, no. 4, pp. 249-267, 2002.

[10] D. E. Merkel and W. L. McGuire, "Ploidy, proliferative activity and prognosis. DNA flow cytometry of solid tumors," Cancer, vol. 65, no. 5, pp. 1194-1205, 1990.

[11] J. S. Ross, "DNA ploidy and cell cycle analysis in cancer diagnosis and prognosis," Oncology, vol. 10, no. 6, pp. 867-887, 1996.

[12] J. Gawrychowski, B. Lackowska, and A. Gabriel, "Prognosis of the surgical treatment of patients with non-small cell lung cancer (NSCLC) - relation to DNA ploidy," European Journal of Cardio-thoracic Surgery, vol. 23, no. 6, pp. 870-877, 2003.

[13] C. Oliani, D. Barana, A. Cazzadori et al., "Cytofluorimetric evaluation of DNA ploidy in lung cancer: a bronchoscopic study," International Journal of Biological Markers, vol. 20, no. 2, pp. 8792, 2005.

[14] V. Ludovini, L. Pistola, V. Gregorc et al., "Biological markers and DNA flow cytometric analysis in radically resected patients with non-small cell lung cancer. A study of the perugia multidisciplinary team for thoracic tumors," Tumori, vol. 94, no. 3, pp. 398-405, 2008.

[15] R. Blanco, C. E. Rengifo, M. Cedeño, M. Frómeta, E. Rengifo, and M. Ramos-Suzarte, "Tumor expression of the carcinoembryonic antigen correlates with high mitotic activity and cell pleomorphism index in lung carcinoma," Journal of Histology, vol. 2013, Article ID 827089, 8 pages, 2013.

[16] D. W. Hedley, M. L. Friedlander, I. W. Taylor, C. A. Rugg, and E. A. Musgrove, "Method for analysis of cellular DNA content of paraffin-embedded pathological material using flow cytometry," Journal of Histochemistry and Cytochemistry, vol. 31, no. 11, pp. 1333-1335, 1983.

[17] J. Vansteenkiste, C. Dooms, and P. de Leyn, "Early stage nonsmall-cell lung cancer: challenges in staging and adjuvant treatment: evidence-based staging," Annals of Oncology, vol. 21, supplement 7, pp. viil89-vii195, 2010.

[18] R.-P. Perng, C.-Y. Chen, G.-C. Chang et al., "Revisit of 1997 TNM staging system-survival analysis of 1112 lung cancer patients in Taiwan," Japanese Journal of Clinical Oncology, vol. 37, no. 1, pp. 9-15, 2007.
[19] X. Jin, Z. Wang, L. Qiu et al., "Potential biomarkers involving IKK/RelA signal in early stage non-small cell lung cancer," Cancer Science, vol. 29, no. 3, pp. 582-589, 2008.

[20] Y. Qiu, H. Yang, H. Chen et al., "Detection of CEA mRNA, p53 and AE1/AE3 in haematoxylin-eosin-negative lymph nodes of early-stage non-small cell lung cancer may improve veracity of $\mathrm{N}$ staging and indicate prognosis," Japanese Journal of Clinical Oncology, vol. 40, no. 2, Article ID hyp144, pp. 146-152, 2010.

[21] I. Petersen, "Chromosomes, ploidy and differentiation of lung cancer," Proceedings of the American Association For Cancer Research, vol. 45, 2004.

[22] D. Choma, J.-P. Daurès, X. Quantin, and J. L. Pujol, “Aneuploidy and prognosis of non-small-cell lung cancer: a meta-analysis of published data," British Journal of Cancer, vol. 85, no. 1, pp. 1422, 2001.

[23] M. Kasprzyk, W. Dyszkiewicz, M. Roszak et al., "DNA ploidy as an independent prognostic factor: 10-year results in a group of patients surgically treated for squamous cell lung cancer," KardIochIrurgIa i TorakochIrurgIa Polska, vol. 3, pp. 327-333, 2012.

[24] H. Hofmann, J. Knolle, H. Bahn, T. Klapperstück, C. Lautenschläger, and H. Neef, "Flow cytometric analysis of DNA content and proliferation and immunohistochemical staining of Ki67 in non-small cell lung cancer," The Journal of Cardiovascular Surgery, vol. 42, no. 4, pp. 555-560, 2001.

[25] W. Dyszkiewicz, M. Kasprzyk, C. Piwkowski, L. Gasiorowski, and R. Ramlau, "The prognostic value of DNA content analysis in patients with squamous cell lung cancer treated surgically," Lung Cancer, vol. 29, no. 3, pp. 161-167, 2000.

[26] H. Otsuka, S. Funai, T. Azumi, S. Hara, K. Okuno, and M. Yasutomi, "Ability of bivariate cytokeratin and deoxyribonucleic acid flow cytometry to determine the biologic aggressiveness of resectable non-small cell lung cancer," Journal of Thoracic and Cardiovascular Surgery, vol. 124, no. 2, pp. 293-298, 2002.

[27] J. T. Ikonen, A. Ojala, J.-P. Salenius, J. Mattila, H. Riekkinen, and T. Wigren, "DNA flow cytometry in surgically treated lung cancer. Prognostic significance," Scandinavian Cardiovascular Journal, vol. 33, no. 4, pp. 228-233, 1999.

[28] T. Visakorpi, K. Holli, and M. Hakama, "High cell proliferation activity determined by DNA flow cytometry and prognosis in epidermoid lung carcinoma," Acta Oncologica, vol. 34, no. 5, pp. 605-609, 1995.

[29] M. M. F. J. Tinnemans, B. Schutte, M.-H. J. H. Lenders, G. P. M. Ten Velde, F. C. S. Ramaekers, and G. H. Blijham, "Cytokinetic analysis of lung cancer by in vivo bromodeoxyuridine labelling," British Journal of Cancer, vol. 67, no. 6, pp. 1217-1222, 1993.

[30] M. Volm, J. Mattern, and J. Sonka, "DNA distribution in nonsmall-cell lung carcinomas and its relationship to clinical behavior," Cytometry, vol. 6, no. 4, pp. 348-356, 1985.

[31] J. Simony, J.-L. Pujol, M. Radal, E. Ursule, F.-B. Michel, and H. Pujol, "In situ evaluation of growth fraction determined by monoclonal antibody Ki-67 and ploidy in surgically resected non-small cell lung cancers," Cancer Research, vol. 50, no. 14, pp. 4382-4387, 1990.

[32] J. A. Alvarez-Riesgo, A. Sampedro, R. Hernandez, M. V. Folgueras, A. Salas- Bustamante, and A. Cueto, "Cell proliferation activity and prognostic index in squamous cell lung carcinoma," Analytical Cellular Pathology, vol. 16, no. 4, pp. 233-242, 1998. 
[33] A. Costa, R. Silvestrini, C. Mochen et al., "P53 expression, DNA ploidy and S-phase cell fraction in operable locally advanced non-small-cell lung cancer," British Journal of Cancer, vol. 73, no. 7, pp. 914-919, 1996.

[34] J. C. Pence, B.-J. M. Kerns, R. K. Dodge, and J. D. Iglehart, "Prognostic significance of the proliferation index in surgically resected non-small-cell lung cancer," Archives of Surgery, vol. 128, no. 12, pp. 1382-1390, 1993. 


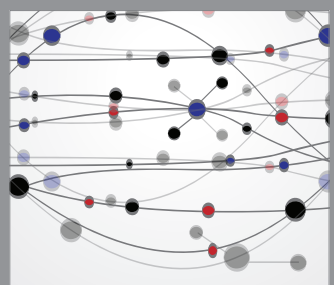

The Scientific World Journal
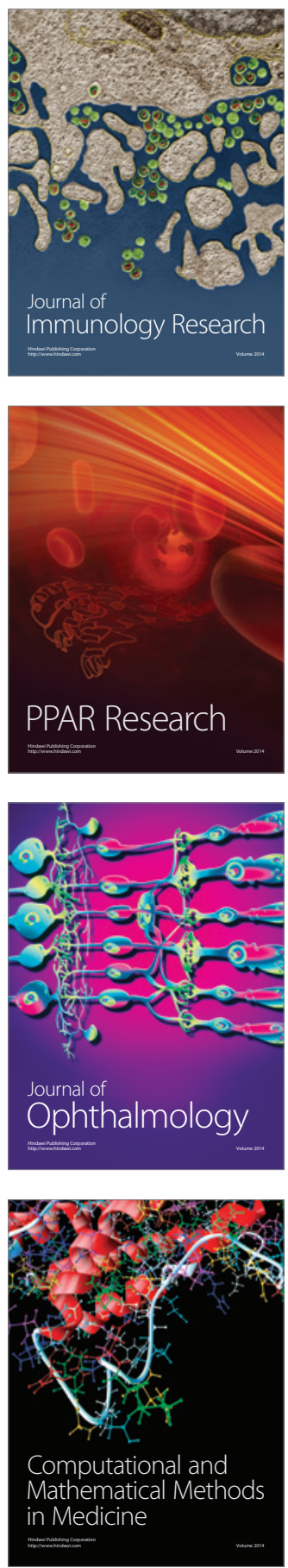

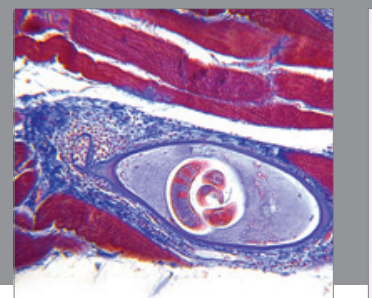

Gastroenterology

Research and Practice
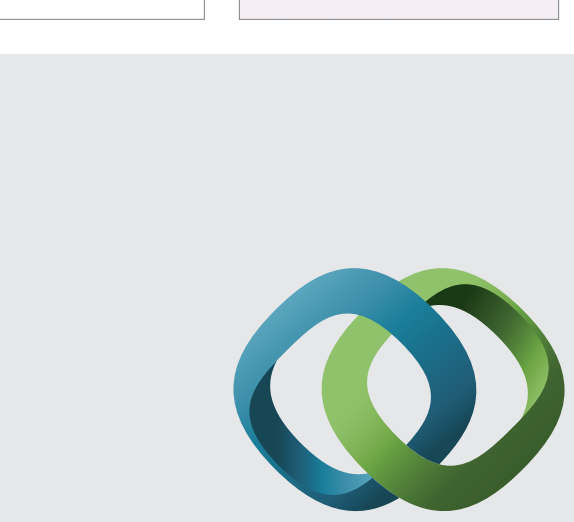

\section{Hindawi}

Submit your manuscripts at

http://www.hindawi.com
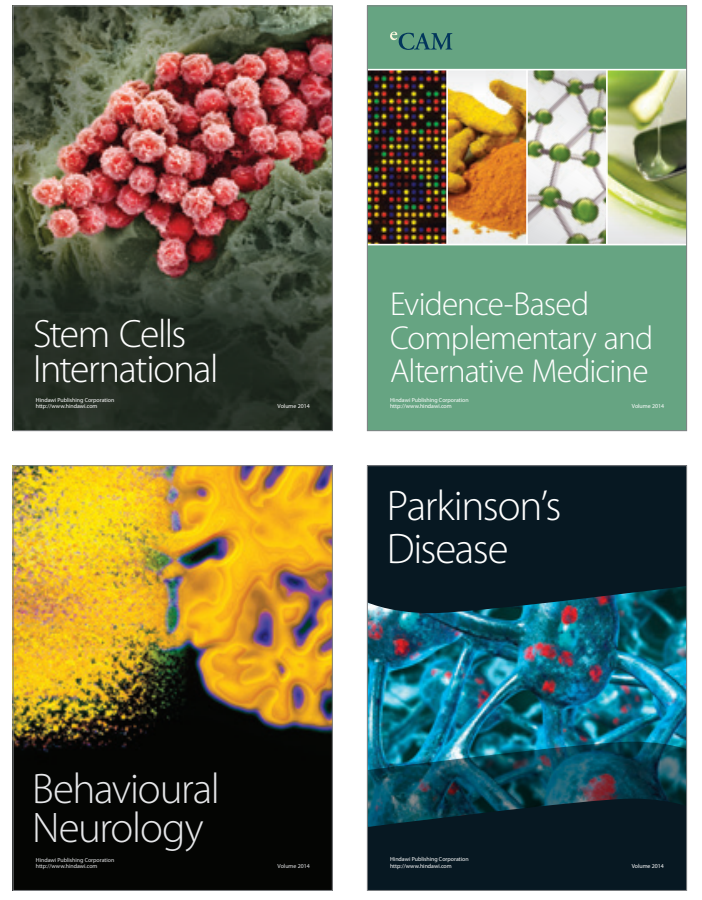
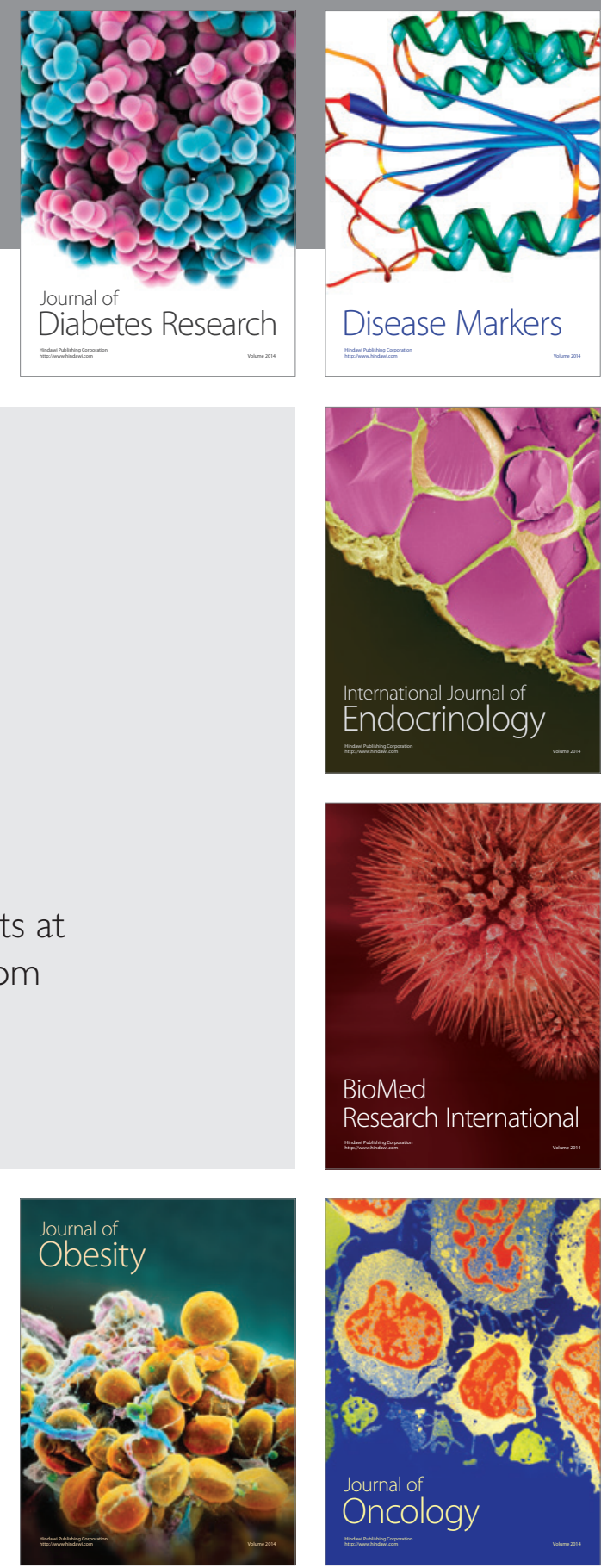

Disease Markers
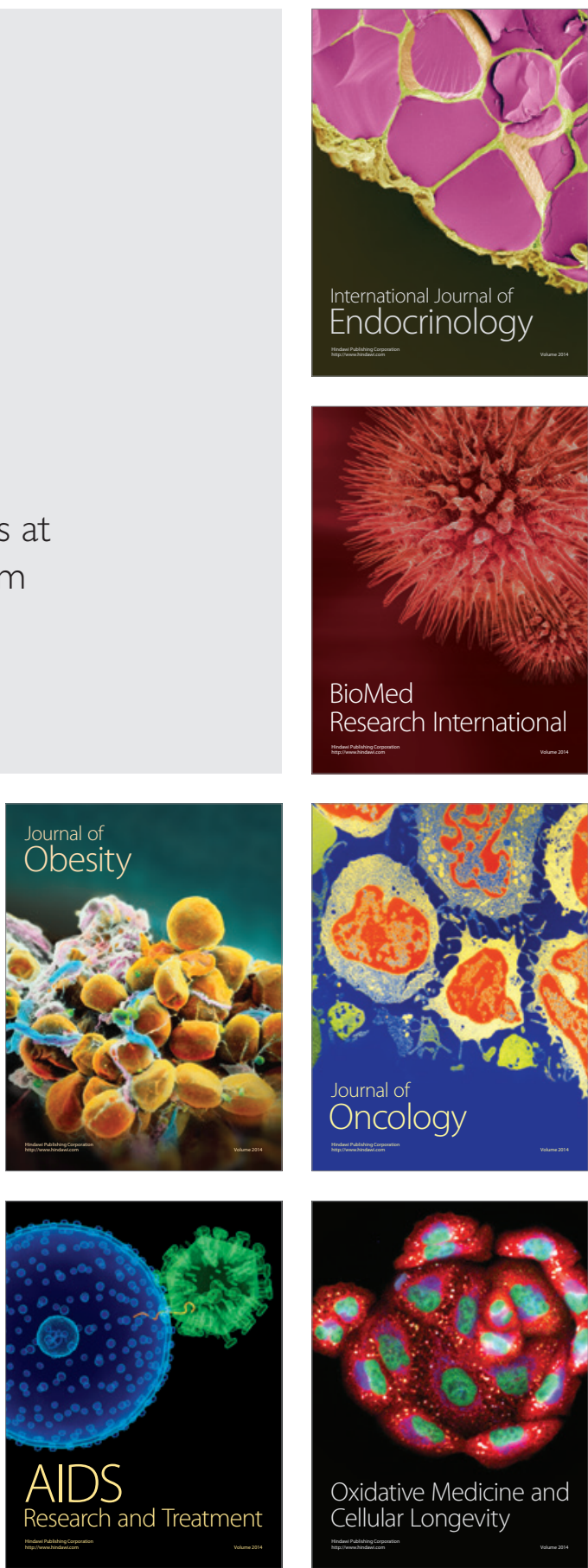